\title{
Macro-aspartate aminotransferase is a possible cause of persistent isolated elevated aspartate aminotransferase level
}

\author{
Karen HK Luk ${ }^{1}$ *, MB, ChB, YT Hui' ${ }^{1}$ MB, BS, Jodis TW Lam¹, MB, BS, KY Lai ${ }^{2}$, MB, BS \\ ${ }^{1}$ Department of Medicine, Queen Elizabeth Hospital, Jordan, Hong Kong \\ ${ }^{2}$ Department of Intensive Care Unit, Queen Elizabeth Hospital, Jordan, Hong Kong \\ *Corresponding author: Ink351@ha.org.hk
}

Hong Kong Med J 2019;25:337-8

https://doi.org/10.12809/hkmj187566

\section{Introduction}

Macroenzymes are formed by complexes of enzymes and their polymerisation products. The enzymes are usually linked to immunoglobulins, proteins, lipoproteins or cell membrane segments. ${ }^{1}$ Therefore, macroenzymes have a higher molecular mass, making their clearance slower and more difficult, and resulting in a spurious increase in serum enzyme concentration. $^{2}$ Macroenzymes are sometimes associated with autoimmune or idiopathic diseases. The prevalence of macroenzymes is variable among different patient groups and not well studied. They are classified into type 1 and type 2 . Type 1 macroenzymes (eg, alanine aminotransferase [ALT], alkaline phosphatase [ALP], amylase, aspartate aminotransferase [AST], gamma glutamyl transferase [GGT], creatine kinase [CK], lactate dehydrogenase $[\mathrm{LDH}]$, and lipase) are formed by enzymes linking to immunoglobulins, mostly immunoglobulin $\mathrm{G}$ and immunoglobulin A. ${ }^{1}$ Type 2 macroenzymes (eg, ALP, amylase, GGT, and CK) are enzyme complexes formed by enzyme polymerisation and their linkage to other serum components such as lipoproteins and cell membrane fragments. ${ }^{1}$ Unlike type 1 macroenzymes, type 2 macroenzymes usually disappear from the circulation following appropriate therapy. Therefore, type 2 macroenzymes are sometimes used as surrogate markers for disease monitoring. ${ }^{1}$

\section{Exemplar case}

We recently experienced the case of a 71-year-old man with asymptomatic isolated increase in serum AST level of $72 \mathrm{IU} / \mathrm{L}$ (normal range: $<40 \mathrm{IU} / \mathrm{L}$ ) found during a scheduled examination for his diabetes, hypertension and non-rheumatic aortic regurgitation at Queen Elizabeth Hospital, Hong Kong, in March 2018. Other parameters of his liver function test were normal.

Investigation was undertaken to look for the cause of the deranged liver function test. Viral hepatitis markers were tested negative for hepatitis $B$ and C. Muscle enzyme CK and LDH levels were normal. Serum copper, ceruloplasmin, iron saturation, and ferritin level were all within normal range. Autoimmune markers including anti-nuclear antibody, anti-mitochondrial antibody, and antismooth muscle antibody were all negative. The immunoglobulin profile was unremarkable. The autoimmune hepatitis score before treatment was 7, which suggested against the diagnosis of autoimmune hepatitis. Ultrasonography of the liver showed several small liver cysts but was otherwise unremarkable. No dilatation of the biliary system was noted. The patient had progressive worsening of serum AST level up to $145 \mathrm{IU} / \mathrm{L}$ on blood tests during the subsequent year. Owing to the largely normal investigation results, absence of symptoms and signs, a diagnosis of macroAST was suspected. Subsequently, polyethylene glycol (PEG) precipitation assay was performed and the diagnosis of macro-AST was confirmed.

\section{Diagnostic pathway}

In our patient, hepatic causes of elevated serum AST level, such as viral hepatitis and autoimmune hepatitis, were unlikely given the negative laboratory tests, normal ultrasound findings, and low autoimmune hepatitis score. Non-hepatic causes of elevated serum AST level include muscle damage and haemolysis. Given the normal muscle enzyme levels (CK, LDH) in our patient, the elevated serum AST level was unlikely attributable to muscle damage. ${ }^{3}$ Patients with macroenzymes are always asymptomatic owing to the falsely elevated concentration of enzymes without an increase in enzymatic activity. Macroenzymes should be suspected when there is a persistent isolated elevated enzyme level without a positive correlation with the other related enzymes; in our patient, serum ALP, ALT and GGT levels were normal.

In our patient, the presence of macroAST was detected by the precipitation technique with PEG. After the patient's serum is treated with PEG, immunoglobulins precipitate out with the immunoglobulin-bound AST, revealing the lower free AST concentration. ${ }^{4}$ Other techniques 
employed to identify the presence of macroenzymes include electrophoresis, immunoinhibition, immunoprecipitation, measurements of heat stability, and chromatography. ${ }^{2,3}$ Typically, a combination of these techniques is used to improve the diagnostic accuracy. ${ }^{3}$

\section{Conclusion}

Despite the rarity of the condition, macro-AST should be suspected when extensive investigations are unable to identify the cause of persistent isolated elevated serum AST level in an otherwise asymptomatic patient. ${ }^{1}$ It should be considered that this is a benign condition and a high index of suspicion may obviate the need for unnecessary extensive investigations. Macroenzymes are associated with not only AST, but also CK, LDH, amylase, and other enzymes.

\section{Author contributions}

All authors had full access to the data, contributed to the study, approved the final version for publication, and take responsibility for its accuracy and integrity.

Concept or design: All authors.

Acquisition of data: All authors.

Analysis or interpretation of data: All authors.

Drafting of the article: KHK Luk, YT Hui.

Critical revision for important intellectual content: All authors.

\section{Conflicts of interest}

All authors have disclosed no conflicts of interest.

\section{Acknowledgement}

Special thanks to Dr Anthony CC Shek, Queen Elizabeth Hospital for the special arrangement for the detection of macro-aspartate aminotransferase in our patient.

\section{Funding/support}

This research received no specific grant from any funding agency in the public, commercial, or not-for-profit sectors.

\section{Ethics approval}

The patient was treated in accordance with the Declaration of Helsinki. The patient provided informed consent for all procedures.

\section{References}

1. Čepelak I, Čvorišćec D. Why is it necessary to recognize macroenzymes? Biochemia Med (Zagreb) 2007;17:52-9.

2. Mbagaya W, Foo J, Luvai A, et al. Persistently raised aspartate aminotransferase (AST) due to macro-AST in a rheumatology clinic. Diagnosis (Berl) 2015;2:137-40.

3. Krishnamurthy S, Korenblat KM, Scott MG. Persistent increase in aspartate aminotransferase in an asymptomatic patient. Clin Chem 2009;55:1573-7.

4. Davidson DF, Watson DJ. Macroenzyme detection by polyethylene glycol precipitation. Ann Clin Biochem 2003;40:514-20.

\section{CORRECTION}

\section{Correction to: Secondary prevention of fragility fractures: instrumental role of a fracture liaison service to tackle the risk of imminent fracture}

In the Figure of this article by Ronald MY Wong et al (Hong Kong Med J 2019;25:235-42, https://doi.org/10.12809/hkmj187593), the word "prevention" was misspelled as "prevdention".

The online article at www.hkmj.org has been corrected. 